\title{
La formation francophone et ses influences sur l'œuvre de Vasile Alecsandri
}

(la dramaturgie)

\section{Cristian Stamatoiu}

\section{OpenEdition}

\section{Journals}

Édition électronique

URL : https://journals.openedition.org/cher/10080

DOI : $10.4000 /$ cher. 10080

ISSN : 2803-5992

\section{Éditeur}

Presses universitaires de Strasbourg

Édition imprimée

Date de publication : 1 décembre 2013

Pagination : 47-57

ISBN : 978-2-86820-560-5

ISSN : 1968-035X

\section{Référence électronique}

Cristian Stamatoiu, «La formation francophone et ses influences sur l'œuvre de Vasile Alecsandri », reCHERches [En ligne], 11 | 2013, mis en ligne le 08 février 2022, consulté le 09 février 2022. URL http://journals.openedition.org/cher/10080 ; DOI : https://doi.org/10.4000/cher.10080

\section{(c) () (ㅇ)}

Ce(tte) œuvre est mise à disposition selon les termes de la Licence Creative Commons Attribution -

Pas d'Utilisation Commerciale - Partage dans les Mêmes Conditions 4.0 International. 


\title{
La formation francophone et ses influences sur l'œuvre de Vasile Alecsandri (la dramaturgie)
}

\author{
Cristian Stamatolu \\ Université de Târgu-Mureş, Roumanie
}

\section{Contexte culturel}

$\mathrm{Au} \mathrm{XIX}{ }^{\mathrm{e}}$, le sous-dialecte moldave - partie intégrante du daco-roumain a participé directement à la formation de la langue roumaine littéraire et de sa littérature. Lors de la modernisation de cet espace, la source des modèles culturels change de manière spectaculaire. Selon l'historien et critique littéraire Eugen Lovinescu'1, disciple de Faguet, L'esprit du siècle (voir Lovinescu 1992: III, 29-35) ne se manifestait plus alors en accord avec la formule: ex Oriente lux, mais ex Occidente lux $x^{2}$. Vasile Alecsandri se trouve précisément à l'origine d'un tel processus concernant la mentalité collective de tous les Roumains.

Soumise à des conditions historiques défavorables, la culture roumaine a survécu longuement à l'intérieur de l'humanisme, ce qui va l'obliger après 1848 à récupérer à la fois, surtout par la filière francophone classicisme et romantisme. Donc, les refondateurs de la littérature roumaine vont se

1 Eugen Lovinescu (31 octobre 1881, Fălticeni, Roumanie - 16 juillet 1943, București, Roumanie) est le père de Monica Lovinescu (19 novembre 1923, București, Roumanie 20 avril 2008, Paris, France), intellectuelle et conscience marquante de l'exile roumain à Paris.

2 Ex Oriente lux/ ex Occidente lux, la lumière (de la culture) parvenue de l'Est/ de l'Occident. Voir Lovinescu: op. cit., I; 17-18). 
manifester vers 1870 dans des circonstances qui les ont menés vers une fusion originale entre des courants artistiques initialement opposés à l'Occident. Ils n'étaient classiques à proprement parler que dans la mesure où ils ont œuvré sur les bases de la littérature roumaine, processus qui se déroule dans notre cas tout au long du $\mathrm{XIX}^{\mathrm{e}}$ siècle. Leur génération comportait en fait des romantiques ayant absorbé des influences classiques (le poète national Mihai Eminescu), des réalistes sous influences passéistes (le prosateur Ioan Slavici, le narrateur Ion Creangă), ou des naturalistes (le dramaturge et prosateur Ion Luca Caragiale) qui cultivaient déjà l'humour de l'absurde (Stamatoiu, 2009: 112-113).

L'apport de la Moldavie entière, des deux rives de la rivière Prut, est définitoire de ce processus car Mihai Eminescu comme Ion Creangă représentent brillamment dans l'universalité l'esprit moldave des Roumains dans sa dimension livresque pour le premier et populaire pour l'autre.

Mais aucun de ces modernes de la littérature roumaine n'aurait pu se manifester pleinement sans la tradition culturelle instaurée en pionnier par Vasile Alecsandri. Animé par un noble esprit de sacrifice, celui-ci s'est proposé de fonder et adapter au contexte roumain les genres et sous-genres littéraires rencontrés dans la culture française. Pleinement assumé, ce programme créatif a communiqué à son œuvre une allure encyclopédique et, parfois une certaine artificialité. Au lieu de se spécialiser dans un ou deux types d'expression littéraire, il a volontairement dispersé son énergie créatrice sur un large horizon: du journalisme à la poésie, de la prose à la dramaturgie, de la réévaluation du folklore jusqu'au management théâtral, dans une multitude des nuances. Il est en fait le premier à avoir établi le prototype de l'intellectuel roumain du XIX ${ }^{\mathrm{e}}$ siècle par ses voyages formateurs en France, par la création d'une tradition francophone/francophile, par la dissémination d'idées progressistes d'origine française, et surtout, par sa création littéraire complexe.

Grâce à la première Union roumaine (entre Moldavie et Valachie en 1859), et après la migration pour raisons politiques du gourou des "classiques", Titu Maiorescu, vers la capitale de Bucarest, le courant initié par Vasile Alecsandri va s'épanouir dans toutes les Principautés de Roumanie. Son succès sera si grand, qu'après l'instauration de la monarchie en 1866 et la Guerre d'Indépendance de 1877, on va assister à une telle popularisation de la francophonie, que celle-ci va même générer des processus pervers dans les rangs des parvenus et des nouveaux riches (voir Id., op. cit.). Ces aberrations socioculturelles ont été la cible d'une ironie manifestée dans la dramaturgie 
de Costache Facca, Vasile Alecsandri et, surtout, du fondateur du théâtre roumain moderne, Ion Luca Caragiale.

Bien sûr, ces réalisations ont été ensuite complétées à l'époque par les classiques de la littérature roumaine introduisant un apport germanique, sous la forme progressiste du Sturm und Drang et d'un romantisme titanesque qui prévoyait d'intégrer aussi l'inspiration du folklore national. Cette filière culturelle a été active surtout en Transylvanie et au nord de la Moldavie (Bucovine) en raison: du prestige culturel allemand dans la zone, de la double formation culturelle (à Berlin, à Paris) de Titu Maiorescu, du prestige moral et de l'action réformatrice du Prince Carol I ${ }^{\text {er }}$ descendant de la famille Hohenzollern.

\section{Vasile Alecsandri - une biographie moldave exemplaire sous le signe culturel de la francophonie}

Vasile Alecsandri est né en $1819^{3}$ dans la famille d'un boyard qui a su valoriser sa fortune dans une ascension sociale spectaculaire ainsi qu'à la faveur d'une formation culturelle audacieuse pour la Moldavie de son temps. Traditionaliste de conceptions orientales, il avait tout de même visité Paris et comme suite de son émancipation culturelle, avait été nommé en 1836 gérant économique de la deuxième troupe théâtrale moderne jouant en roumain: «Conservatorul filarmonic - dramatic ${ }^{4}$ de Iassy, ancienne capitale de la Moldavie.

Dans ce contexte favorable, le jeune Vasile Alecsandri a bénéficié d'une instruction solide acquise en dépit d'un enseignement public encore à ses débuts. Il a commencé ses études primaires selon la mode dans les familles aisées du temps, c'est-à-dire à domicile, sous la tutelle d'un précepteur: le moine Gherman Vida. En 1828 il est inscrit à Iassy comme élève interne au Pensionnat de garçons de Victor Cuénim où on enseignait le français. Là, il se lie d'amitié avec des élèves tels que les futurs hommes politiques progressistes Alexandru Ioan Cuza, premier souverain des Principautés Unies de Roumanie $^{5}$ (1859-1866), Mihail Kogalniceanu, homme politique voué

3 Ses biographes accréditent plusieurs dates pour sa naissance, an allant de 1819 à 1821 , selon les documents utilisés.

4 Conservatorul filarmonic - dramatic [Le Conservatoire philarmonique et dramatique].

5 L'union des Roumains dans l'état moderne de "la Grande Roumanie» a eu lieu le $1^{\text {er }}$ décembre 1918, à la suite de la Grande Guerre durant laquelle le Corps Expéditionnaire Français dirigé par le général Berthelot a contribué de manière décisive aux victoires de l'Armée Roumaine. 
à l'Unification, l'Indépendance et généralement, au progrès de la culture roumaine.

Avec ces compagnons et quelques autres, il part en août 1834 vers Paris où le groupe «des soldats du futur» (Nicolescu G. C. 1962: 24) arrive après 21 jours (!) de pénible voyage. Ils seront accueillis par Filip Furnaraki dans sa maison de la rue Notre Dame des Champs et il rencontre d'autres jeunes Roumains venus dans le même but de Bucarest (Valachie) dont Ion Ghica, le plus important de tous.

S'intéressant aux études de Vasile Alecsandri, Furnaraki décide de l'envoyer auprès d'un professeur privé pour qu'il puisse passer son baccalauréat selon les exigences de la loi française sur l'enseignement. Le baccalauréat en lettres obtenu en octobre 1835, Alecsandri hésite entre plusieurs possibilités: étudier le droit (qu'il trouve trop aride), la médecine (les dissections à la morgue lui répugnent) ou même la chimie (dans les laboratoires de Gaulthier de Glaubry il est intoxiqué à la suite d'une explosion arsenicale). Pour finir, il convainc son père, venu en visite à Paris, de lui permettre de devenir ingénieur en constructions civiles. Mais pour s'inscrire dans cette Faculté il avait besoin d'un baccalauréat ès sciences: il s'adresse à cette fin au professeur Cotte et en vue de renforcer son niveau en sciences exactes au professeur Bonin. Cette nouvelle tentative a un résultat négatif et, déçu, le jeune Alecsandri s'abandonne à sa passion: la littérature. Il étudie la littérature française spécialement guidé par M. Cotte devenu son ami, fréquente les spectacles de théâtre pour assister aux comédies à la mode de Scribe et aux drames de Victor Hugo, visite grands musées et galeries d'art où se prépare la révolution impressionniste, se laisse emporter au concert par la musique de Chopin et commence à écrire en français des poèmes (ainsi un madrigal pour conquérir le cœur de son premier amour, la nièce du général Lassalle!). De même, il prend désormais l'habitude de rédiger ses lettres en français, surtout sa correspondance avec ses amis francophones ${ }^{6}$. À cette époque, il préfère une vie plus libre et déménage $n^{\circ} 8$ rue Racine, chez Charles de Lapeyrouse: sa mère et lui formeront sa nouvelle famille française. Pour Alecsandri désormais c'est l'époque dorée de fréquentation des cafés: le Café Corneille et le Café Procope rassemblant les jeunes intellectuels d'Europe autour des idées des grands esprits du moment: Hugo, Fauriel, Quinet, Herder, Lamartine... (Nicolescu, 1962: 25-39).

6 Des fragments de cette ample correspondance ont été utilisés pour l'illustration de l'air du temps par George Călinescu dans son ouvrage biographique: Vasile Alecsandri [Vasile Alecsandri], București, Editura Tineretului, 1965. 
C'est dans ce contexte qu'arrive de Moldavie en 1839 un message capital pour sa destinée: il doit revenir à Iassy où l'attend un avenir brillant. Son père refuse de continuer à subventionner son dolce farniente à Paris, considérant la culture déjà acquise par son fils comme largement suffisante pour l'activité réformatrice qui l'attend. Mais afin que la chute du Paradis parisien soit acceptée sans protestation, on organise pour lui un retour digne d'un dandy. Accompagné de ses amis Nicolae Docan et Costache Negri, il revient en Moldavie par un trajet touristique et culturel incluant l'Italie: Paris-Lyon-Marseille-Livourne-Florence-Rome. De là, il revient à Florence pour enchaîner sur: Bologne-Padoue-Venise-Trieste-Vienne (Ghitulescu, 1979: 15), où le banquier paternel le fait monter de force sur un bateau appareillant pour le bas Danube.

Après les effusions du retour, Vasile Alecsandri va s'impliquer dans la modernisation de son pays commencée par des grands boyards qu'intéresse le capitalisme - non révolutionnaire. Il est d'abord nommé de 1840 à 1842 co-directeur du théâtre de Iassy avec son ami Mihail Kogalniceanu et l'écrivain Costache Negruzzi. Ils ont pour tâche d'organiser cette institution culturelle d'allure occidentale de manière interculturelle viable. La troupe roumaine devait être refondée sous la direction de Costache Caragiale ${ }^{7}$. Elle devait fonctionner aux côtés de la troupe française des frères Fourreaux et de la troupe d'opéra allemande de Madame Terez Frich. Le but principal était la création d'un répertoire dramatique roumain original suivant le Programme esthétique publié le $1^{\mathrm{er}}$ mai 1840 dans le premier numéro de la Dacia Literară, revue éditée par un collectif - dirigé par Mihail Kogălniceanudont Alecsandri faisait aussi partie. Dans ce contexte culturel, il en appelle naturellement à sa culture francophone, écrit de savoureuses comédies, des vaudevilles à finalité éducative à la manière d'Eugène Scribe. Il s'agit de comédies de l'imposture et du quiproquo: Cinovnicul și modista (Le petit employé et la modiste), Farmazonul din Hîrlău (Le Franc-Mazon (sic!) de Hîrlăus) et en dehors du directorat: Iorgu de la Sadagura (Iorgou de Sadagura), Iașii în carnaval (La Ville de Iassy en carnaval) et beaucoup d'autres après 1850 .

7 Il s'agit ici de l'oncle paternel du fondateur du théâtre roumain moderne, I. L. Caragiale.

$8 \mathrm{Au}$ lieu du terme "franc-maçon", l'auteur utilise ici ironiquement pour dénoncer l'imposture une forme archaïque et déviante de ce néologisme français; conformément aux principes de l'orthographe actuelle, dans le cas de toponymes comme «Hîrlău» on n'autorise pas l'utilisation de «â" à l'intérieur du mot, ce qui nous oblige d'utiliser pour le même son: «î̀. 
Il publie aussi à cette époque des poèmes d'inspiration folklorique roumaine, des vers en français et à la française dans une revue francophone locale conduite par l'intellectuel illuministe Gheorghe Asachi: Le glaneur moldo-valaque. Il connaît un grand amour avec Elena Negri. Elle devient la muse à qui il dédie le poème Steluța (La petite étoile). Mais deux ans plus tard, une affection de poitrine éteint son étoile adorée, ce qui provoquera en lui une blessure profonde. A la suite de cette tragédie personnelle, il s'orientera vers une lyrique intimiste, un peu précieuse dont le premier volume: Doine și lăcrămioare (Des chansons t des muguets) sera publié à Paris en 1835. Le titre le plus représentatif de ce recueil est Dri-dri, appellatif dont il gratifiait naguère sa bien-aimée (Cornea, 1967: XXXVI-XL).

Impliqué en 1848 dans la rédaction du Manifeste révolutionnaire des «bonjouristes", il se réfugie à Paris après une escale en Transylvanie qui dure jusqu'à la fin de 1849. Il y revient en 1854-1855 pour visiter l'Exposition universelle. Exilé politique, Alecsandri fréquente des rédactions de la presse libérale où il rencontre Jules Michelet ou Edgar Quinet qui devient un ami très proche. Ces événements ouvrent un nouvel horizon au poète inspiré par les valeurs des Loges révolutionnaires. Il commence à écrire des poèmes militants qui vont influencer l'histoire de son pays. Il transplante l'esprit hugolien en contexte politique moldave et en langage poétique d'inspiration folklorique. Le Poème "Hora Unirii» démultiplié comme un manifeste sur feuilles volantes a influencé l'Union entre Moldavie et Valachie de janvier 1859 sous le signe de la double élection du Prince légendaire Alexandru Ioan Cuza. En fait, Alecsandri avait été nommé initialement futur Prince unificateur mais dans sa noblesse et sa modestie, il avait décliné la proposition. L'Union réalisée, il est envoyé en mission diplomatique sensible en France (en qualité de Ministre des Affaires etrangères). Il est reçu par Napoléon III, par le comte Walewski ministre de l'Extérieur, il rencontre son idole Lamartine. Il se rend en Angleterre dans le Piémont, obtenant la reconnaissance diplomatique de son pays unifié dans des conditions inespérées.

Après cet intermède historique, Vasile Alecsandri resté le même boyard à l'esprit moldave ne s'exprimera plus que comme porte-parole culturel et politique des Roumains : créant le texte de l'hymne national ${ }^{9}$ utilisé de 1947 à la chute du Rideau de Fer. Ce patriotisme ne l'a pas empêché de souffrir comme un Français (sa génération est celle de Rimbaud) de la

9 Trăiască regele! [Vive le Roi!]. 
chute de la Commune... Il entend bien prendre sa revanche sur les cercles réactionnaires européens en 1877 quand le Principat roumain obtient son indépendance d'Etat. Il écrit donc un journal poématique sur les batailles victorieuses de la jeune armée roumaine contre les Ottomans. Aussitôt publiés dans les journaux, ces textes ont élevé le moral de la Nation pendant la Guerre d'Indépendance. Un volume intitulé Ostașii nostri (Nos soldats) les a rassemblés. La même année, la Société pour l'étude des langues romanes de Montpellier lui décerne un prix pour Cântecul gintei latine (Le Chant latin), (Platon, 1980: 201-202).

Déçu par des rivalités politiques, Alecsandri fait de longues retraites dans son domaine de Mircești. Cet esprit polyvalent a été le grand préromantique qui a su élever la littérature roumaine jusqu'à un niveau d'acceptation de la révolution romantique, de la démocratie bourgeoise, du développement réaliste plus tardif. Génie de la littérature roumaine, il est élu en 1867 comme membre de la future Académie (la «Société littéraire») aux côtés de jeune rival et successeur spirituel Mihail Eminescu. Celui-ci le décrira dans Epigonii (Les épigones) comme: «un roi de la poésie/ Eternellement jeune et heureux» (Eminescu, 1978: 29). Alecsandri s'est illustré en 1868 comme le créateur du cycle lyrique Pasteluri (Pastels) qui dépeint les quatre saisons d'une nature roumaine pittoresque. Comme dans l'esprit romantique lamartinien, le cadre naturel entre en résonance avec l'état d'âme d'un promeneur solitaire très semblable en attitude au jeune et romantique Stendhal.

L'autre «chez moi» de Alecsandri est naturellement la France où il fait des voyages privés en 1866, 1869-1870 et fait la connaissance sur la riviera de Prosper Mérimée. En 1882 en Provence, il rencontre aussi le poète Mistral.

De retour à Paris en 1885, il est ambassadeur de son idéal de vie. Plusieurs années, il lui sera de plus en plus difficile de faire la navette entre Paris et Mircești. Il est en effet atteint du cancer qui causera sa fin, avant son entrée au Panthéon de la littérature roumaine, le 22 août 1890 (Cornea, op.cit.: XLI-LIV). 
Les faux amis du français et le comique de langage dans la dramaturgie de Vasile Alecsandri

Sa dramaturgie de jeunesse a une savoureuse continuation après 1850, quand il bâtit l'épopée comique de son personnage "Coana Chirita» (Mère Chiritza ${ }^{10}$ ), aux gaffes toujours proverbiales.

Parallèlement, selon le modèle de Hugo et de Shakespeare, il écrira des drames historiques. Deux sont des méditations dramatiques sur la destinée de l'écrivain génial parvenu à l'âge de la maturité comme Fântâna Blanduziei (La Fontaine de la Nostalgie), 1883, sur la vie d'Horace et Ovidiu (Ovide), 1884, sur l'exil d'Ovidius Publius Naso au bord de la mer Noire. Une autre Despot Vodă (Le despote) (1880) est inspiré d'un fait insolite de l'histoire féodale moldave. IL propose un traitement romantique de l'ascension sociale d'un personnage exceptionnel manipulant les trois âges de l'éternel féminin. Ce trait le rapproche du modèle hugolien d'Hernani et de Ruy Blas (joué en 1837 lors du premier séjour de Alecsandri à Paris). Alecsandri a aussi écrit une comédie en français, intitulée: Les bonnets de la comtesse.

Dans Coana Chirița, le dramaturge a fustigé l'imposture des parvenus et nouveaux riches surgissant après une révolution. Pour jouer les gens de qualité, ces personnages prétendaient parler le français aussi bien que s'ils l'avaient pratiqué pendant leurs études. Cette prétention les démasquera face au public roumain hautement francophone, capable de sanctionner l'usage des "faux amis", les fausses étymologies, en bref la précarité sociale de «l'homme récent» du temps (Patapievici, 2002: 108-132). La cible de cette satire était moins les francophones véritables que les "franco-aphones" (Stamatoiu, 2009:1-2) dont l'expression générait des situations linguistiques d'un immense humour involontaire.

La comédie Chirita în provincie (1852) montre une disgracieuse et vénale créature incarnant ce qu'elle croit être une sylphide émancipée à la française (Alecsandri, 1977:V, 411-478). Elle a apporté de Paris, outre de nouvelles habitudes (fumer, pratiquer l'équitation) et des robes ridiculement somptueuses, un professeur chargé d'apprendre le français à Guliță, son fils stupide qu'elle croit génial. L'acte I, scène 3 montre que sa grande ambition est d'en faire un érudit. Elle explique un tel idéal à Monsieur Charles, qu'elle appelle à la moldave: «monsiu Şarlă». Sa prononciation fautive lui fait

10 La comédiographie de Chirița comprend les vaudevilles: Chirița în Iaşi [Kiritza à Iassy] et Chirița în provincie [Kiritza en province], aussi comme les comédies: Chirița în voiaj [Kiritza en voyage], Chirița în balon [Kiritza en ballon]. 
utiliser une forme corrompue de provenance populaire pour "Monsieur» $\left(\right.$ monsiu $\left.{ }^{11}\right)$ quand pour le prénom "Charles», elle fait appel à une fausse étymologie, le superposant au terme populaire «şarlăă ${ }^{12} » q u i$ signifie aussi: chien paresseux, bon à rien. C'est ce parasite qu'elle doit payer pour satisfaire son caprice de mode. Avec la même ironie, l'auteur transcrira le nom de ce professeur suivant la prononciation de Chirița, obtenant la forme originale: Şarl! (Alecsandri, 1977: V, 411-422).

Le personnage principal veut absolument se faire entendre par Şarl mais en usant de barbarismes résultant d'une traduction mot à mot du moldave en français de quelques expressions idiomatiques connues dans tout l'espace roumain. La première est: " $a$ fi tobă de carte $^{13}$ », syntagme qui signifie par périphrase "être érudit». L'intellectuel est si gonflé de connaissances que sa peau devrait être tendue comme celle d'un tambour. Rêvant du brillant avenir de son fils, Chirita émet l'hypothèse qu'il devienne «tambour d'instruction", ce qui horripile le malheureux Français. Elle voudrait que son fils parle couramment cette langue. Mais pour formuler ce vou, elle opère un calque de l'expression: « $a$ vorbi ca apă $»^{14}$ : parler comme l'eau, couramment. Aussi poli qu'un Français, le professeur est prêt à faire un compliment empoisonné: il souhaite à Gulita de parler un jour le français aussi bien que sa propre mère.

Chirita ne veut pas que son fils perde son temps, c'est-à-dire qu'il le perde pour "des fleurs de coucou», selon une autre expression changée en barbarisme («de florile cucului»). Elle lui demande par conséquent de traduire des mots choisis à l'improviste: «friptură » (grillade), «furculiță » (fourchette), «învîrtită ${ }^{15}$ » (danse). Dans son ignorance du sens de ces termes, il improvise bêtement, ajoutant à la racine roumaine du mot une terminaison très française. Mais sa mère accueille avec enthousiasme les monstruosités linguistiques: «fripturision», «furculision », «învârtision»!

11 Pour la conformité phonétique, ici il faut le lire exactement comme on l'écrit.

12 Dans le sous-dialecte moldave on prononce le « $\breve{A}$ » final sous une forme spécifique, plus fermé, le «ÎI», fait qui rend cette prononciation identique par chance avec celle du nom propre français. Il faut aussi tenir compte que la transcription parfaite du groupe « $\mathrm{CH}$ » du français c'est dans le roumain le «Ş».

13 A fi - être; tobă, n.f. - tambour; de carte - à cause des livres (lus).

14 A vorbi - parler; ca pe apă - comme sur l'eau.

15 Friptură, n.f. - rôti; Furculiță, n.f. - fourchette; Învârtită n.f. - danse folklorique roumaine dont les figures principales sont les pirouettes des couples. 
Le professeur exaspéré contre-attaque en usant du même moyen. Il use d'un terme populaire roumain appris récemment: "gogoman » (imbécile) qui ressemble beaucoup au terme français "gogo». Il lui ajoute la terminaison trouvée par Gulita, obtenant l'hybride: «Gogomantion, va!».

Un tel comique de langage sera exploité par les comédies du successeur le plus important de Vasile Alecsandri: I.L.Caragiale, dont les expressions sont toujours d'actualité. Cependant la popularité d'Alecsandri a survécu jusqu'à nos jours puisqu'on entend au quotidien des calembours forgés par lui. Si quelqu'un fait l'erreur de traduire mot à mot en français ou une autre langue une expression idiomatique roumaine, il est sanctionné de ce verdict: «furculision!» De même, si l'on veut ridiculiser quelqu'un pour son cosmopolitisme, on peut le réduire à néant en l'appelant: «monsiu».

De tels faits de langage montrent que l'œuvre d'Alecsandri est toujours actuelle. La seule différence est qu'aujourd'hui, la vague de l'imposture soulève une inculture portée par les anglicismes.

\section{Bibliographie}

Alecsandri V., 1977, Teatru, [Théatre], vol. 5, București, Minerva.

Călinescu G., 1965, Vasile Alecsandri [Vasile Alecsandri], Bucureşti, Editura Tineretului.

Cornea P., 1969, «Prefață, Tabel cronologic şi Notă» [Préface, Table chronologique et Note], dans: Vasile Alecsandri, Doine şi lărcămioare [Des doïnas et des mugets], Bucureşti, Editura pentru Literatură, collection «Biblioteca pentru toți», p. V-LV.

Eminescu M., 1978, Epigonii [Les Epigones], dans Poezii - Proză literară [Poèmes Prose littéraire] vol. I., Bucureşti, Cartea românească.

Ghițulescu E., 1979, Vasile Alecsandri [Vasile Alecsandri], Bucureşti, Editura Albatros, collection «Monografii».

Lovinescu E., 1992, Istoria civilizației româneşti [L'Histoire de la civilisation roumaine] (1924-1925), III vol., Bucureşti, Editura BPT.

Nicolescu G.C., 1962, Viața lui Vasile Alecsandri, [La Vie de Vasile Alecsandri], Bucureşti, Editura pentru Literatură.

Patapievici H.-R., 2002, Omul recent [L'Homme récent], Bucureşti, Humanitas.

Platon M., 1980, Vasile Alecsandri, poeții Felibri şi «Cântecul Gintei latine» (O pagină din istoria relațiilor culturale franco-române), [Vasile Alecsandri, les poètes Félibriges et La Chanson du latin (une page des relations culturelles francoroumaines)], avec un résumé en français par Maria Platon, Iaşi, Junimea. 
Resplandy F., 2006, (consultants pour le roumain: Cristian Stamatoiu et Sanda Rîpeanu), L'étonnant voyage des mots français dans les langues étrangères, Paris, Bartillat.

Stamatoiu C., 2009, «Le Suprême absurde ionescien: traduire les comédies de Caragiale en français», Symbolon, ${ }^{\circ}$ 17, p. 112-124.

Stamatoiu C., 2009, «Les trouvailles francophones des personnages franco-aphones de la comédiographie de Caragiale. «Une nuit orageuse» (I, 4 et 7)», Conférence Internationale de l'Université «Petru Maior», Tîrgu Mureş, 2009, IEIM 3, publié sur le CD dans: Documentele IEIM 3 [Les Documnets de IEIM 3], Tîrgu Mureş, Universitatea «Petru Maior». 